\title{
Original
}

\section{Predictive modeling of the hypothalamic-pituitary-adrenal (HPA) axis response to acute and chronic stress}

\author{
Vladimir M. Marković ${ }^{1)}$, Željko Čupić ${ }^{2)}$, Vladana Vukojevićs) and Ljiljana Kolar-Anić ${ }^{1)}$ \\ 1) Faculty of Physical Chemistry, University of Belgrade, 11000 Belgrade, Serbia \\ 2) Institute of Chemistry, Technology and Metallurgy, University of Belgrade, Department of Catalysis and Chemical Engineering, \\ 11000 Belgrade, Serbia \\ 3) Department of Clinical Neuroscience, Karolinska Institutet, 17176 Stockholm, Sweden
}

\begin{abstract}
Detailed dynamics of the hypothalamic-pituitary-adrenal (HPA) axis is complex, depending on the individual metabolic load of an organism, its current status (healthy/ill, circadian phase (day/night), ultradian phase) and environmental impact. Therefore, it is difficult to compare the HPA axis activity between different individuals or draw unequivocal conclusions about the overall status of the HPA axis in an individual using single time-point measurements of cortisol levels. The aim of this study is to identify parameters that enable us to compare different dynamic states of the HPA axis and use them to investigate self-regulation mechanisms in the HPA axis under acute and chronic stress. In this regard, a fourdimensional stoichiometric model of the HPA axis was used. Acute stress was modeled by inducing an abrupt change in cortisol level during the course of numerical integration, whereas chronic stress was modeled by changing the mean stationary state concentrations of CRH. Effects of acute stress intensity, duration and time of onset with respect to the ultradian amplitude, ultradian phase and the circadian phase of the perturbed oscillation were studied in detail. Bifurcation analysis was used to predict the response of the HPA axis to chronic stress. Model predictions were compared with experimental findings reported in the literature and relevance for pharmacotherapy with glucocorticoids was discussed.
\end{abstract}

Key words: HPA axis, Stress, Cortisol, Predictive modeling, Circadian and ultradian rhythmicity

OSCILLATORY dynamics appears to be essential for self-organization and self-regulation in living systems. Oscillations with different periodicity, from millisecond to annual range, are implemented at all levels of organization of living organisms, from the molecular to the biosphere level, occurring in processes as diverse as gene transcription in the cell nuclei to seasonal migrations of species between ecosystems [1, 2]. The hypothalamic-pituitary-adrenal (HPA) axis, a neuroendocrine system involved in maintaining homeostasis in mammalian organisms under physiological conditions and stress [3-5], is no exception to that rule. Cortisol, the HPA axis principal hormone in humans, exhibits complex dynamic behavior with two char-

Submitted: May 21, 2011; Accepted Aug. 2, 2011 as EJ11-0037 Released online in J-STAGE as advance publication Aug. 18, 2011 Correspondence to: Vladana Vukojević, Department of Clinical Neuroscience, Karolinska Institutet, CMM L8:01, 17176 Stockholm, Sweden. E-mail: vladana.vukojevic@ki.se Ljiljana Kolar-Anić, Faculty of Physical Chemistry, University of Belgrade, Studentski trg 12-16, 11000 Belgrade, Serbia.

E-mail: 1kolar@ffh.bg.ac.rs acteristic frequencies: ultradian oscillations, with a period of 20-120 min [4-6] superimposed on circadian oscillations, with a period of about $24 \mathrm{~h}$. The importance of circadian rhythms for adequate functioning of the HPA axis has been recognized for years $[7,8]$; in addition, new experimental [9-15] and theoretical [16-27] results offer enough evidence to support the indispensable roles of ultradian oscillatory dynamics of HPA hormones levels for normal physiology. Since ultradian and circadian oscillations operate on different time scales, their effects are manifested in different biological realms. However, being coupled they seem to contribute synergistically to better integration and adaptation of an organism to the unpredictable dynamic environment.

Adequate dynamics of HPA activity is essential for maintaining homeostasis in mammalian organisms. Changes in the detailed dynamics of the HPA axis emerge routinely while the axis copes with a myriad of external stimuli $[5,28]$. At the same time, the overall dynamics of the HPA axis is remarkably robust and stable in an organism [29, 30]. Stress and a number of 
Table 1 A basic model describing self-regulation in the HPA system in humans.

\begin{tabular}{|c|c|c|c|}
\hline$\stackrel{\mathrm{k}_{0}}{\longrightarrow} \mathrm{CRH}$ & $r_{0}=k_{0}$ & $\mathrm{k}_{0}=1.82556 \times 10^{-8} \mathrm{M} \mathrm{min}^{-1}$ & (R1) \\
\hline$\stackrel{\mathrm{k}_{\mathrm{m}}}{\longrightarrow}$ ALDO & $r_{m}=k_{m}$ & $\mathrm{k}_{\mathrm{m}}=6.0852 \times 10^{-11} \mathrm{M} \mathrm{min}^{-1}$ & (R2) \\
\hline $\mathrm{CRH} \stackrel{\mathrm{k}_{1}}{\longrightarrow} \mathrm{ACTH}$ & $r_{1}=k_{1}[C R H]$ & $\mathrm{k}_{1}=1.82556 \mathrm{~min}^{-1}$ & (R3) \\
\hline $\mathrm{ACTH} \stackrel{\mathrm{k}_{2}}{\longrightarrow} \mathrm{CORT}$ & $r_{2}=k_{2}[A C T H]$ & $\mathrm{k}_{2}=3.6 \times 10^{-2} \mathrm{~min}^{-1}$ & (R4) \\
\hline $\mathrm{ACTH} \stackrel{\mathrm{k}_{3}}{\longrightarrow} \mathrm{ALDO}$ & $r_{3}=k_{3}[\mathrm{ACTH}]$ & $\mathrm{k}_{3}=2.88 \times 10^{-4} \mathrm{~min}^{-1}$ & (R5) \\
\hline $\mathrm{ACTH}+2 \mathrm{CORT} \stackrel{\mathrm{k}_{4}}{\longrightarrow} 3 \mathrm{CORT}$ & $r_{4}=k_{4}[\mathrm{ACTH}][\mathrm{CORT}]^{2}$ & $\mathrm{k}_{4}=1.26 \times 10^{14} \mathrm{~min}^{-1} \mathrm{M}^{-2}$ & (R6) \\
\hline$A L D O+2$ CORT $\stackrel{k_{5}}{\longrightarrow}$ CORT & $r_{5}=k_{5}[A L D O][C O R T]^{2}$ & $\mathrm{k}_{5}=7.0524 \times 10^{12} \mathrm{~min}^{-1} \mathrm{M}^{-2}$ & (R7) \\
\hline $\mathrm{ACTH} \stackrel{\mathrm{k}_{6}}{\longrightarrow} \mathrm{P}_{1}$ & $r_{6}=k_{6}[A C T H]$ & $\mathrm{k}_{6}=5.346 \times 10^{-2} \mathrm{~min}^{-1}$ & (R8) \\
\hline $\mathrm{CORT} \stackrel{\mathrm{k}_{7}}{\longrightarrow} \mathrm{P}_{2}$ & $r_{0}=k_{7}[$ CORT $]$ & $\mathrm{k}_{7}=4.0986 \times 10^{-1} \mathrm{~min}^{-1}$ & (R9) \\
\hline
\end{tabular}

The reactions, presented in the form of simple chemical reactions, epitomize complex biochemical pathways. $\mathrm{P}_{1}$ and $\mathrm{P}_{2}$ stand for products of ACTH and cortisol elimination, respectively. Reaction rates, derived by the mass action law, are indicated in the second column. The third column contains corresponding rate constants. The rate constants were selected so that the numerical results veritably emulate the in vivo dynamics of the HPA axis. For example, rate constants $\mathrm{k}_{6}$ and $\mathrm{k}_{7}$ were selected in such a way that ACTH and cortisol half-lives agree well with experimentally determined values reported in the literature. The concentration is expressed in $\mathrm{M}=\mathrm{mol} \cdot \mathrm{dm}^{-3}$.

illnesses are associated with short- or long-term perturbations of the HPA dynamics, changing the amplitude and/or frequency of HPA hormones discharge and their mean levels. Such changes are observed in many diseases: primary (Addison's disease) and secondary adrenocortical insufficiency, Cushing's syndrome, visceral obesity, diabetes, hypertension, osteoporosis and major depression [4, 31].

The aim of this study is to understand how the HPA system adapts to external stimuli by achieving altered dynamic states, and what the possible consequences of the altered HPA axis dynamics are. To this aim, we use mathematical modeling, numerical simulations and dynamical systems theory approaches to investigate the dynamic behavior of the HPA system under acute and chronic perturbance, i.e. stress. To enable a meaningful comparison between different dynamic states, we first define new parameters that characterize these states and use them to investigate self-regulation mechanisms in the HPA axis under acute and chronic stress. We compare the model predictions with experimental observations reported in the literature. Furthermore, we use this analysis to discuss in the Appendix how this new understanding may be practically applied for designing treatment with glucocorticoids. These immunosuppressive drugs, commonly used for acute or chronic treatment of inflammatory diseases [32], may perturb the HPA axis dynamics thereby causing unwanted side-effects.

\section{The HPA model description}

The HPA axis dynamics was emulated using a fourdimensional stoichiometric model presented in Table 1. This low-dimensional model, described in detail in our previous studies [17-19], comprises CRH (corticotropin-releasing hormone), ACTH (adrenocorticotropic hormone), ALDO (aldosterone) and CORT (cortisol) as dynamic variables. Reactions (R1)-(R9) epitomize the following complex pathways: (R1) describes basal CRH production from the hypothalamic paraventricular nucleus; (R2) describes aldosterone production under the renin-angiotensin system control; (R3) describes the CRH stimulated ACTH production from the pituitary; (R4) and (R5) describe ACTH stimulated production of cortisol and aldosterone from the adrenal cortex; (R6) describes the positive feedback actions of cortisol, acting through hippocampal GR to enhance $\mathrm{CRH}$, and consequently ACTH and its own production; (R7) exemplifies cortisol negative feedback through hippocampal MR where both aldosterone and cortisol compete for the same receptor, as well as through hypothalamic and pituitary GR; (R8) and (R9) describe ACTH and cortisol elimination, respectively. Thus, ultradian self-regulation in the model is achieved through the experimentally established positive and negative feedback effects of cortisol on the HPA system via glucocorticoid (GR) and mineralocorticoid receptors (MR) [33-36]. However, these receptors are not included directly, but rather introduced implicitly 
through reactions (R6) and (R7).

The ultradian dynamics of the HPA system is described by the following set of ordinary differential equations, obtained from the equations given in Table 1 in accordance with the law of mass action [37, 38]:

$$
\begin{aligned}
& \frac{\mathrm{d}[\mathrm{CRH}]}{\mathrm{dt}}=\mathrm{k}_{0}-\mathrm{k}_{1}[\mathrm{CRH}] \\
& \begin{aligned}
\frac{\mathrm{d}[\mathrm{ACTH}]}{\mathrm{dt}}=\mathrm{k}_{1}[\mathrm{CRH}]-\left(\mathrm{k}_{2}+\mathrm{k}_{3}+\mathrm{k}_{6}\right)[\mathrm{ACTH}] \\
-\mathrm{k}_{4}[\mathrm{ACTH}][\mathrm{CORT}]^{2}
\end{aligned} \\
& \begin{aligned}
\frac{d[A L D O]}{d t}=k_{m}+k_{3}[A C T H] & -k_{5}[A L D O][C O R T]^{2}
\end{aligned} \\
& \begin{aligned}
\frac{d[C O R T]}{d t}= & k_{2}[\text { ACTH }]+k_{4}[\text { ACTH }][\text { CORT }]^{2} \\
& -k_{5}[\text { ALDO }][\text { CORT }]^{2}-k_{7}[\text { CORT }] .
\end{aligned}
\end{aligned}
$$

[CRH], [ACTH], [ALDO] and [CORT] stand for the concentration of $\mathrm{CRH}, \mathrm{ACTH}$, aldosterone and cortisol, respectively.

To account for the circadian rhythm governed CRH production, an extrinsic periodic function $(D)$ has been introduced:

$$
\begin{gathered}
D=\mathrm{d}_{1}-0.079145093 \cdot \mathrm{d}_{2}+\{0.064 \cdot \sin (2 \pi t / 1440)+ \\
0.12 \cdot \operatorname{abs}[\sin (\pi t / 1440)]\} \cdot \mathrm{d}_{2} .
\end{gathered}
$$

This function emulates well the asymmetry of the 24 $\mathrm{h}$ rhythm in humans, with the nocturnal phase lasting 8 hours (Strictly speaking, the function $D$ is not differentiable at $\mathrm{t}=\mathrm{v} \cdot 1440, \mathrm{v}=1,2 \ldots$ However, this function is continuous and no problems were encountered during numerical integration). It affects the inflow rate of $\mathrm{CRH}$ into the system, transforming $\mathrm{k}_{0}$ in Eq. (1) into $\mathrm{k}_{D}(\mathrm{t})=\mathrm{k}_{0} \cdot D$. The multiplier $D$ couples the rate constant of CRH production $\left(\mathrm{k}_{0}\right)$ to extrinsic circadian regulation (Fig. 1c and d). Consequently, CRH evolution changes from monotonic (Fig. 1a) to oscillatory (Fig. 1c), and the dynamics of cortisol ultradian oscillations becomes more complex (Fig. 1b compared to Fig. 1d). Parameters $d_{1}$ and $d_{2}$ in Eq. (5) decouple the mean daily CRH level in the hypothalamic-pituitary portal vessels (governed by $\mathrm{d}_{1}$ ) from the amplitude of the circadian CRH oscillation (governed by $\mathrm{d}_{2}$ ). The very nature of function $D$ is not altered by the parameter separation - in essence, it is the same function as was used before $[17-19,25]$. However, separation of the parameters enables us to investigate the effect of mean daily $\mathrm{CRH}$ levels in the hypothalamic-pituitary portal vessels independently from the effect of daily CRH amplitude on the HPA axis dynamics, thereby facilitating the study of individual differences (it is well-established that circadian dynamics under basal conditions is very stable for a person, but can differ significantly between individuals). Daily oscillations in CRH levels, driven by the endogenous circadian clock have been modeled differently by different authors (e.g. [16]).

\section{Methods}

\section{Computational methods}

Numerical simulations were employed to emulate different acute/chronic stress conditions and test the validity of the model's response to such simulation designs. In order to find solutions of the set of ordinary differential equations (Table 1), the Gear algorithm [39] for integration of stiff differential equations was used.

\section{Definition of dynamic variables and perturbation parameters}

A key feature of the model presented in Table 1 is the spontaneous evolution of ultradian oscillations, arising as a consequence of an intrinsic instability in the model [17-19, 25]. Thus, ultradian oscillations occur under defined conditions, without the need for circadian rhythm driven CRH pulse generation [17-19]. This very important characteristic of the model is in agreement with experimental findings showing that ultradian rhythmicity of cortisol does not cease after surgically disconnecting the hypothalamus from the pituitary [40], leaving the pituitary corticotrophs unstimulated by the hypothalamic CRH.

A practical consequence of this feature of the model is that without circadian oscillations, the stationary state concentration of CRH ([CRH $]_{\mathrm{SS}}$ ) is a control parameter defined by the ratio of rate constants for CRH production $\left(\mathrm{k}_{0}\right)$ and consumption $\left(\mathrm{k}_{1}\right)$ [19]:

$$
[\mathrm{CRH}]_{\mathrm{SS}}=\frac{\mathrm{k}_{0}}{\mathrm{k}_{1}} \text {. }
$$

Thus, the mean level of $\mathrm{CRH}$, the frequency and the amplitude of ultradian oscillations are constant once a non-equilibrium steady state is being reached (Fig. 1a and $1 b)$.

Circadian regulation of hormone concentrations leads to more complex dynamic behavior, yielding their variable daily levels (illustrated for CRH and cortisol in Fig. 1c and 1d, respectively). Under such conditions, the mean concentration of the hormones, and 

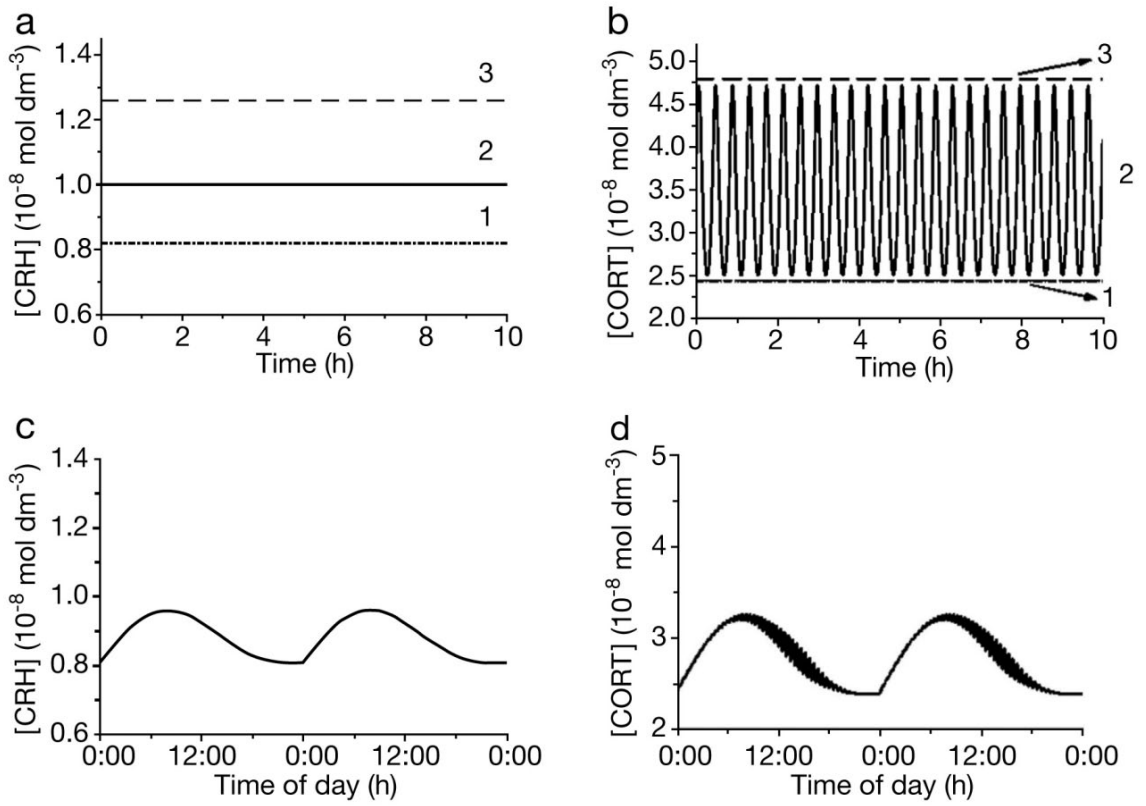

Fig. 1 Temporal evolution of ultradian CRH (a) and cortisol (b) concentrations. Different cortisol dynamics is observed for different basal levels of $[\mathrm{CRH}]_{\mathrm{SS}}$. (1) At low CRH levels, $[\mathrm{CRH}]_{\mathrm{SS}}=0.8 \cdot 10^{-8} \mathrm{~mol} \mathrm{dm}^{-3}$, a low cortisol concentration steady state is observed, $[\mathrm{CORT}]_{\mathrm{SS}}=2.49 \cdot 10^{-8} \mathrm{~mol} \mathrm{dm}{ }^{-3}$. (2) When $[\mathrm{CRH}]_{\mathrm{SS}}=1.0 \cdot 10^{-8} \mathrm{~mol} \mathrm{dm}^{-3}$, cortisol exhibits ultradian oscillations. The amplitude of ultradian cortisol oscillations is $A(C O R T)=4.054 \cdot 10^{-8} \mathrm{~mol} \mathrm{dm}^{-3}$ and the frequency $v_{\mathrm{u}}=2.40 \mathrm{~h}^{-1}$. (3) At high CRH levels, $[\mathrm{CRH}]_{\mathrm{SS}}=1.3 \cdot 10^{-8} \mathrm{~mol} \mathrm{dm}{ }^{-3}$, a high cortisol concentration steady state is observed, $[\mathrm{CORT}]_{\mathrm{SS}}=4.79 \cdot 10^{-8} \mathrm{~mol} \mathrm{dm}{ }^{-3}$. A full bifurcation diagram is presented in Fig. 5a. Temporal evolution of CRH (c) and cortisol (d) concentrations under ultradian and circadian control. Under the effect of the circadian rhythm emulating function $D, D=\mathrm{d}_{1}-0.079145093 \cdot \mathrm{d}_{2}+\{0.064 \cdot \sin (2 \pi$ $t / 1440)+0.12 \cdot \operatorname{abs}[\sin (\pi t / 1440)]\} \cdot d_{2}$, CRH exhibits circadian oscillations, while cortisol exhibits both ultradian and circadian oscillations, with ultradian oscillations being superimposed on the circadian. The initial values of the hormone species $\mathrm{CRH}$, ACTH, aldosterone and cortisol: $[\mathrm{CRH}]_{0}=0.9 \cdot 10^{-8} \mathrm{~mol} \mathrm{dm}^{-3},[\mathrm{ACTH}]_{0}=8.5 \cdot 10^{-8} \mathrm{~mol} \mathrm{dm}{ }^{-3},[\mathrm{ALDO}]_{0}=1.6 \cdot 10^{-8} \mathrm{~mol} \mathrm{dm}^{-3}$ and $[\mathrm{CORT}]_{0}=2.8 \cdot 10^{-8} \mathrm{~mol} \mathrm{dm}{ }^{-3}$, respectively, were used for all numerical simulations in this paper. For basal physiological conditions, $D$ function parameters are taken throughout the paper to be as follows: $\mathrm{d}_{1}=0.88524$ (corresponds to $<$ [CRH] $>=$ $0.88524 \cdot 10^{-8} \mathrm{~mol} \mathrm{dm}{ }^{-3}$, see Eq. 7) and $\mathrm{d}_{2}=0.957$.

the frequencies and amplitudes of ultradian oscillations are no longer constant, but depend on the actual state of the system. Therefore, absolute values of perturbation intensity cannot be compared directly. In order to make a meaningful comparison between different dynamic states possible, we first define new variables and perturbation parameters that take into account the daily variability in HPA hormonal levels.

The mean daily concentration of $C R H$ in the hypothalamic-pituitary portal vessels $(<[C R H]>)$. Under circadian regulation, the rate of $\mathrm{CRH}$ production changes periodically over time, modeled through the effect of function $D$ on $\mathrm{k}_{0}$. Consequently, [CRH] ss (Eq. 6) is no longer constant and becomes a variable whose value repeats itself after $24 \mathrm{~h}$ (Fig. 1c). In order to quantify the average daily CRH levels in the hypothalamic-pituitary portal vessels, the mean daily concentration of $\mathrm{CRH}$ in the hypothalamic-pituitary portal vessels during $24 \mathrm{~h}$ is introduced:
$<[\mathrm{CRH}]>=\frac{<\mathrm{k}_{\mathrm{D}}(\mathrm{t})>}{\mathrm{k}_{1}}=\frac{\mathrm{k}_{0} \cdot<\mathrm{D}>}{\mathrm{k}_{1}}=\frac{\mathrm{k}_{0} \cdot \mathrm{d}_{1}}{\mathrm{k}_{1}}$,

where $<D>$ is the average value of the function $D$ over $24 \mathrm{~h}$.

The absolute amplitude $\left(A_{i}(\mathrm{X})\right)$. Under the circadian drive, each two successive ultradian oscillations are characterized by different amplitudes (Fig. 1d). Therefore, the simple definition of amplitude as the difference between two successive extreme values is not satisfactory for further use. Hence, the absolute amplitude of the $i$-th ultradian oscillation of species $\mathrm{X}\left(A_{i}(\mathrm{X})\right)$ was defined as a difference between the concentration maximum $\left([\mathrm{X}]_{\mathrm{max}, i}\right)$ and the arithmetic mean of concentration values of the two nearest minima, before $\left([\mathrm{X}]_{\min -b e f o r e, i}\right)$ and after $\left([\mathrm{X}]_{\min -a f t e r, i}\right)$ the considered maximum:

$$
A(X)=[X]_{\max , i}-\frac{[X]_{\min -\text { before }, i}+[X]_{\text {min-after }, i}}{2} .
$$


The relative amplitude $\left(A_{\mathrm{rel}}\right)$. The relative amplitude $\left(A_{\text {rel }}\right)$ of species $\mathrm{X}$ is defined as the ratio of amplitudes of a selected reference oscillation after $\left(A_{\text {ref }}(\mathrm{X})\right)$ and before $\left(A_{\text {ref }, 0}(X)\right)$ a perturbation:

$$
A_{r e l}=\frac{A_{\text {eff }}(X)}{A_{r e f, 0}(X)} \text {. }
$$

Thus, when $A_{\text {rel }}<1, A_{\text {rel }}>1$ or $A_{\text {rel }}=1$, the amplitude of the reference oscillation has decreased, increased or has not changed, respectively.

In connection to the relative amplitude of the reference oscillation, it is convenient to define three reference values of perturbator $P$ designated as $[P]_{\text {low, }},[P]_{\text {high }}$ and $[P]_{\min }$. The perturbator can be an internal species (ACTH, CRH, CORT or ALDO) or an externally introduced species that interacts with the internal species. In this study, cortisol usually played this role. $[P]_{\min }$ is the concentration of perturbator $\mathrm{P}$ for which the relative amplitude $A_{\text {rel }}$ reaches its minimal value. $[P]_{\text {low }}$ is the lowest concentration of perturbator $\mathrm{P}$ that induces a visible effect on the HPA system's dynamics (arbitrarily taken to change the amplitude of the reference oscillation for more than $\pm 2.5 \%)$. $[P]_{\text {high }}$ is a non-zero concentration of perturbator $\mathrm{P}$ for which $A_{\text {rel }}=1$. As we shall see later, these values enable us to efficiently compare different dynamic states.

The ultradian and circadian phase angles. The HPA model response to a perturbation is sensitive to the phase angle at which the perturbation is applied. Two phase angles, one with respect to the period of the i-th ultradian oscillation $\left(T_{\mathrm{u}, \mathrm{i}}\right)$ and the other with respect to circadian period $\mathrm{T}_{\mathrm{C}}\left(\mathrm{T}_{\mathrm{C}}=24 \mathrm{~h}\right)$ were therefore defined.

The ultradian phase angle $\left(\varphi_{\mathrm{u}}\right)$. If the ultradian period is the time between two successive minima surrounding the $\mathrm{i}$-th ultradian maximum $\left(t_{\mathrm{u}, \min _{2}, i}-\right.$ $\left.t_{\mathrm{u}, \min _{1}, i}\right)$, the ultradian phase angle $\left(\varphi_{\mathrm{u}}\right)$ represents the time passed from the time-point of the first minimum of a perturbed i-th ultradian oscillation $\left(t_{\mathrm{u}, \min _{1}, i}\right)$ to the time-point of perturbation $\left(t_{\mathrm{u}, \mathrm{p}, i}\right),\left(t_{\mathrm{u}, \mathrm{p}, i}-t_{\mathrm{u}, \mathrm{min}_{1}, i}\right)$, relative to the time-period of ultradian oscillation $\left(T_{\mathrm{u}, i}\right)$ :

$$
\varphi_{u}=\frac{t_{u, p, i}-t_{u, \min _{1}, i}}{T_{u, i}}=\frac{t_{u, p, i}-t_{u, \min _{1}, i}}{t_{u, \min _{2}, i}-t_{u, \min _{1}, i}},
$$
mum.

where $t_{\mathrm{u}, \min _{2}, i}$ is the time-point of its second mini-

The circadian phase angle $\left(\varphi_{\mathrm{c}}\right)$. The circadian phase angle $\left(\varphi_{c}\right)$ represents the time passed from the time-point of the first minimum of a perturbed circadian oscillation $\left(t_{\mathrm{c}, \mathrm{min}, 1}\right)$ to the time-point of perturba- tor's application $\left(t_{\mathrm{c}, \mathrm{p}}\right)$ relative to the time-period of circadian oscillation $\left(\mathrm{T}_{\mathrm{c}}\right)$ :

$$
\varphi_{c}=\frac{t_{c, p}-t_{c, \min , 1}}{T_{c}} \text {. }
$$

\section{Results}

An important distinguishing characteristic of stress is its duration. Acute stress is usually defined as an abrupt, short-lasting (seconds to hours timescale) and isolated perturbation, whereas chronic stress is recurring, persisting for several hours a day for weeks, months or longer [41]. Bearing in mind these differences, acute or chronic stress need to be modeled accordingly.

\section{Acute stress}

Acute stress was simulated in the form of a singlepulse perturbation, meaning that the computer program stops the integration algorithm at a given time-point, momentarily rising the concentration of the perturbed species for the given amount. The integration proceeded using as initial conditions the new concentration of the perturbing agent, leaving the concentration of other intermediates unaltered and equal to their values just before the algorithm was stopped. Response of the model to perturbations with chief stress hormones, $\mathrm{CRH}$ or cortisol, were studied. Both species generated analogous response in the HPA axis. Therefore, we presented here results for perturbations with cortisol only. For comparison with CRH, see references [17-19].

\section{Effect of acute stress intensity and the ultradian phase angle}

In the HPA model with ultradian oscillations alone, the response of the HPA system to stress depends critically on the perturbation intensity and the phase angle of the perturbed ultradian oscillation. A detailed analysis of this case can be found in Refs. 17 and 18.

\section{Effect of acute stress intensity and the circadian phase angle}

Similar, but somewhat more complex behavior was observed for the model comprising both circadian and ultradian oscillations (Figs. 2 and 3). A stressful stimulus of the same intensity can increase or decrease the amplitude of ultradian oscillations, depending on the phase angle of the reference ultradian oscillation at 

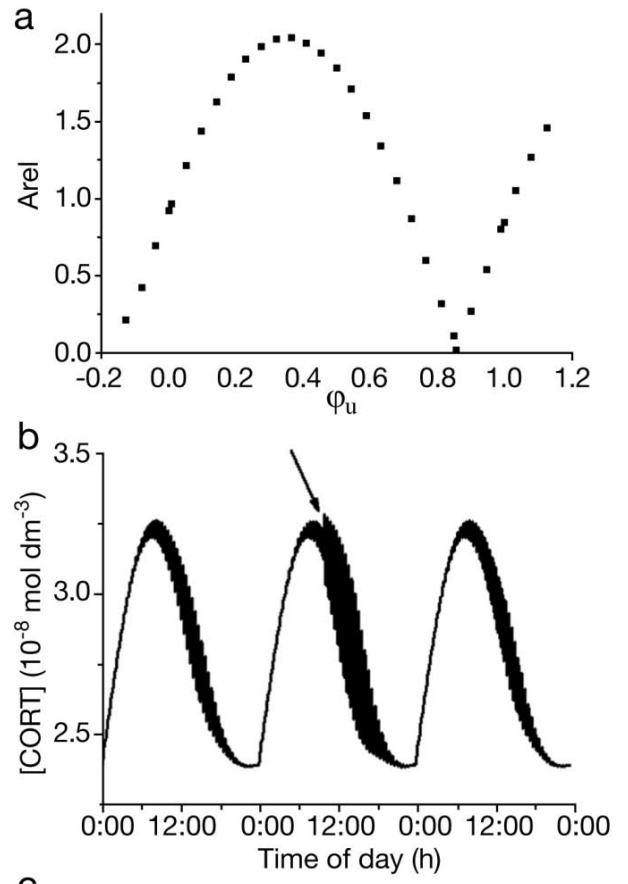

C
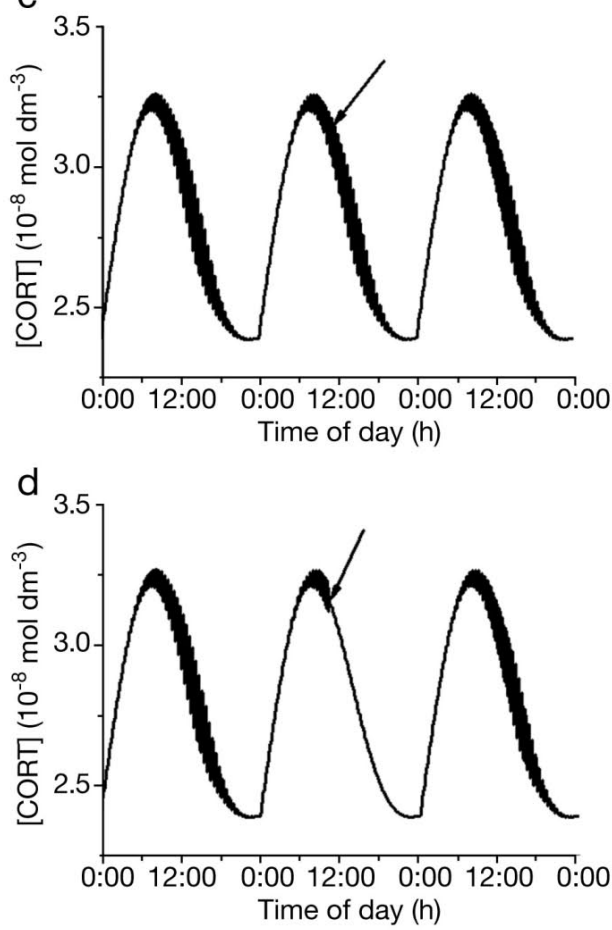

Fig.2 (a) Response of the HPA system to cortisol concentration perturbation of the same intensity ([CORT] $=0.45 \cdot 10^{-9}$ mol $\mathrm{dm}^{-3}$ ) elicited at different ultradian phase angles $\varphi_{\mathrm{u}}$. The response of the system to perturbations is expressed by the relative amplitude $\left(A_{\text {rel }}\right)$ (see Eq. 9). Time-series of cortisol concentration illustrate three qualitatively different responses of the HPA system. (b) The amplitude of the ultradian oscillations increases upon a perturbation induced at $\varphi_{u}=0.365$. (c) The amplitude of ultradian oscillations does not change upon a perturbation induced at $\varphi_{\mathrm{u}}=$ 0.700 . (d) The amplitude of ultradian oscillations decreases markedly upon a perturbation induced at $\varphi_{\mathrm{u}}=0.857$.
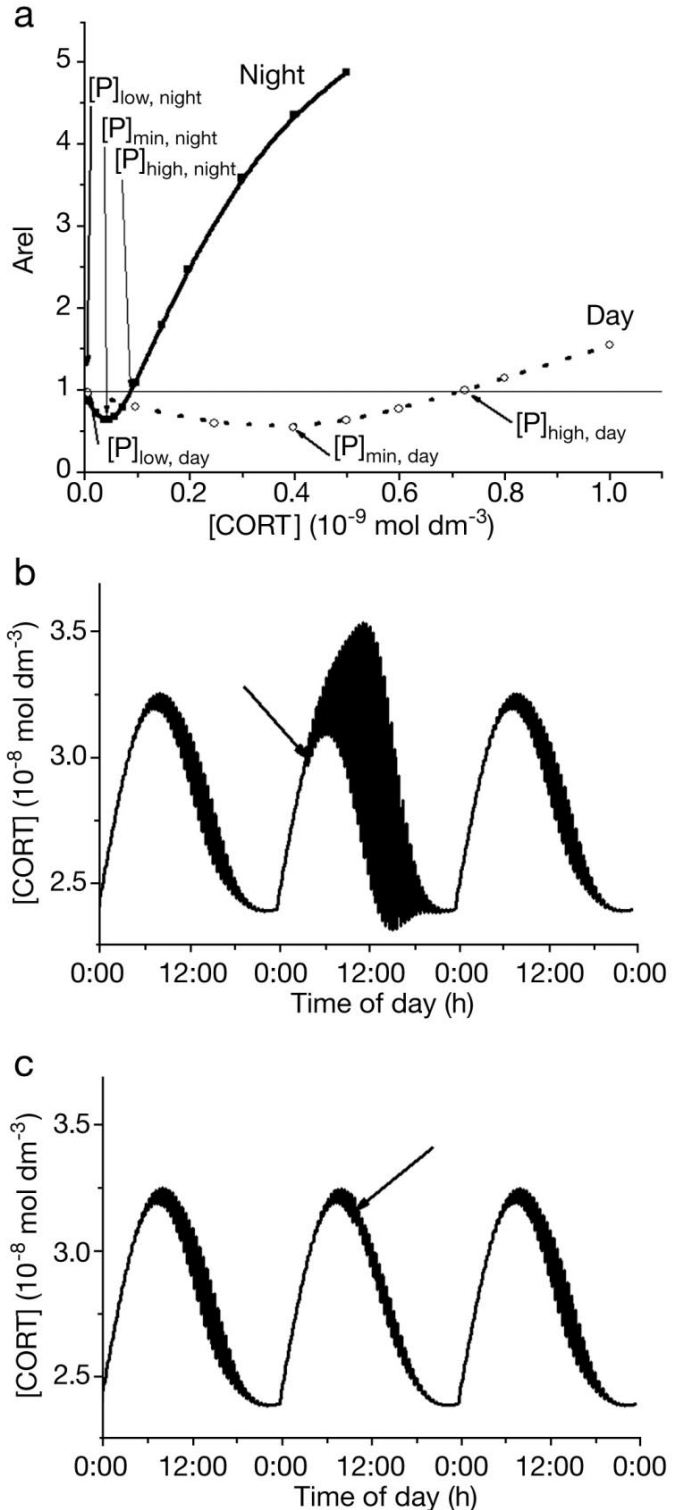

Fig. 3 The HPA model response to acute stress elicited during the diurnal/nocturnal phase of the day. In all cases, perturbations are applied at the same ultradian phase angle $\left(\varphi_{\mathrm{u}}=0.766\right)$. (a) Relative cortisol amplitude $\left(A_{\text {rel }}\right)$ as a function of the exogenous cortisol concentration pulse ([CORT]). The perturbations are applied at night, $\varphi_{\mathrm{c}}=$ $0.168(\square)$, and daytime, $\varphi_{C}=0.428(\circ)$. The horizontal line $A_{\text {rel }}=1$ divides the graphic area into two regions, where the amplitude of the ensuing oscillation is decreased $\left(A_{\text {rel }}\right.$ $<1)$ or increased $\left(A_{\text {rel }}>1\right)$ in response to acute stress. An intersection between the line $A_{\text {rel }}=1$ and the curve $A_{\text {rel }}=$ $\mathrm{f}([\mathrm{CORT}])$ corresponds to a point at which the concentration of the perturbator does not change the relative amplitude of cortisol. Down-arrows indicate the values ([P] $]_{\text {low,night }}$, $\left.[P]_{\text {high,night }},[P]_{\text {min,night }}\right)$ at night, whereas up-arrows denote the corresponding values during daytime $\left([P]_{\text {low,day }}\right.$, $\left.[P]_{\text {high,day, }}[P]_{\text {min,day }}\right)$. Temporal evolution of cortisol after a perturbation pulse of exogenous cortisol $\left(0.4 \cdot 10^{-9}\right.$ mol $\mathrm{dm}^{-3}$ ) applied at the ascending, night-time (b) and descending, daytime (c) phase of the circadian rhythm. 

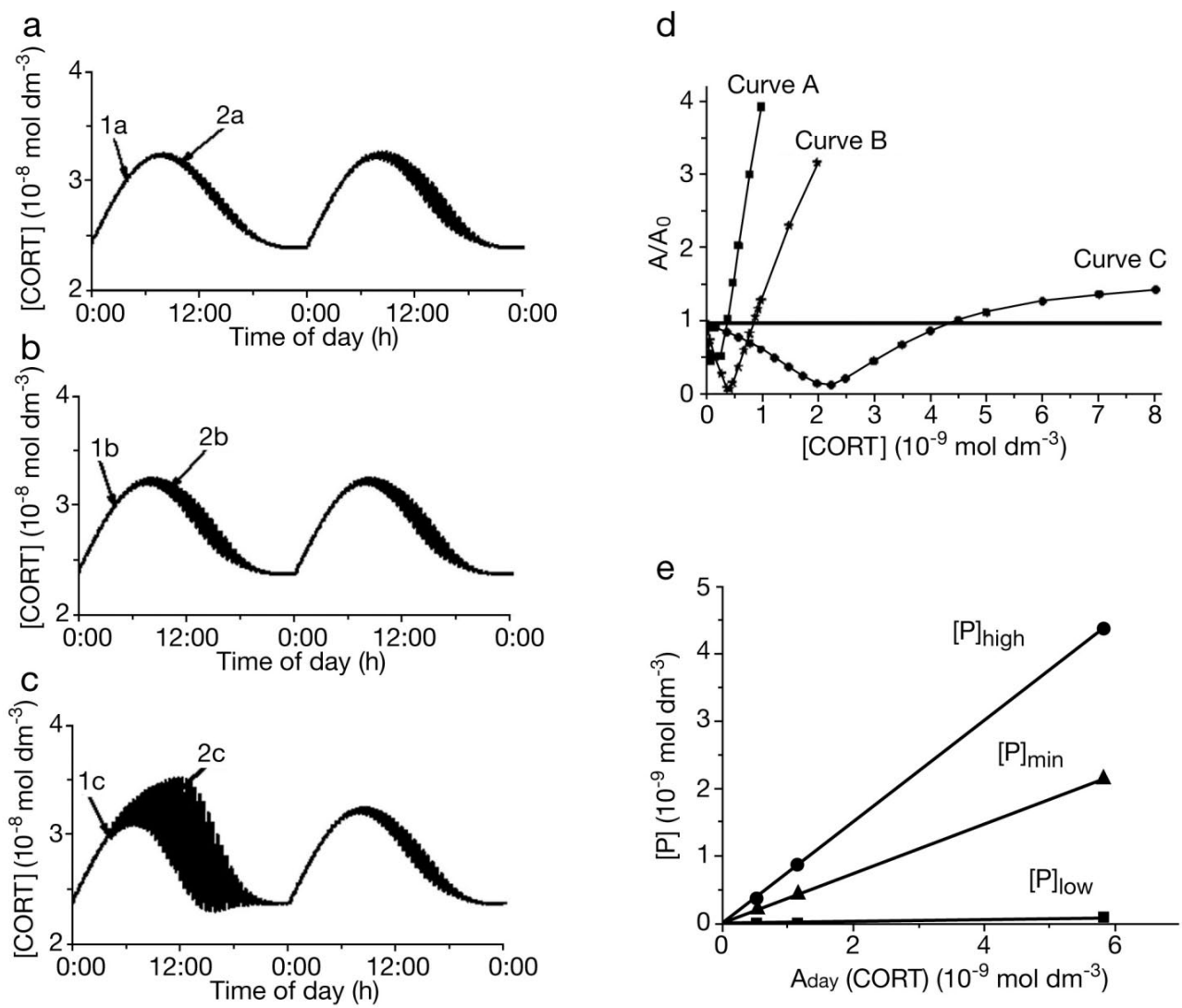

Fig. 4 Response of the HPA axis model to two successive acute perturbations with cortisol. The first perturbation, indicated by arrows $1 \mathrm{a}, 1 \mathrm{~b}$ and $1 \mathrm{c}$, is applied at the same ultradian phase angle $\left(\varphi_{u}=0.766\right)$, during the ascending circadian phase (night for humans, around 04:00). Depending on the intensity of the first perturbation, the response of the HPA system is different: (a) a decrease in CORT amplitude is observed for acute perturbation with [CORT] $=0.075 \cdot 10^{-9} \mathrm{~mol} \mathrm{dm}^{-3}$; (b) no perturbation, [CORT] $=0 \mathrm{~mol}$ $\mathrm{dm}^{-3}$; (c) increase in CORT amplitude is observed for acute perturbation with [CORT] $=0.4 \cdot 10^{-9} \mathrm{~mol} \mathrm{dm}^{-3}$. Thus, three distinct dynamical states (a, b, c) are induced, characterized with different ultradian dynamics. The new dynamical states, characterized by different ultradian oscillation amplitudes $\left(A_{\text {day }}(C O R T)\right.$ ) at daytime (for humans, around 10:00): $A_{\text {day }}^{\text {a }}(C O R T)=0.5190 \cdot 10^{-9}$ mol dm ${ }^{-3}, A_{\text {day }}^{b}(C O R T)=1.1445 \cdot 10^{-9} \mathrm{~mol} \mathrm{dm}^{-3}$ and $A_{\text {day }}^{c}(C O R T)=5.8210 \cdot 10^{-9} \mathrm{~mol} \mathrm{dm}^{-3}$ are perturbed once again, using series of different cortisol concentrations but always at the same ultradian phase angle $\left(\varphi_{\mathrm{u}}=0.850\right)$. These secondary perturbations,

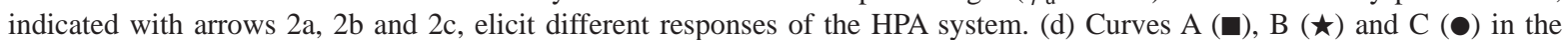
comparative diagram describe differential responses of the HPA system's different initial states a, b, c to described cortisol pulse designs (2a, 2b, 2c), respectively. (e) The sensitivity parameters $[P]_{\text {low }},[P]_{\text {high }}$ and $[P]_{\min }$ depend linearly on the amplitude of the dynamical states $(a, b, c)$ that are established after the first perturbation.

which the stress was induced (Fig. 2).

The HPA system response to perturbations depends also on the circadian phase (Fig. 3). Fig. 3a shows the response of the HPA system to cortisol perturbations of different intensities elicited during the day- and nighttime. The HPA system response was displayed as a function of relative amplitudes of the reference oscillation $\left(A_{\text {rel }}\right)$ versus the concentration of the exogenous cortisol pulse ([CORT]), $A_{\text {rel }}=\mathrm{f}$ ([CORT]). Perturbations were applied at the same ultradian phase angle $\left(\varphi_{\mathrm{u}}=0.766\right)$. Numerical simulations showed that the HPA system's response to stress induced during the night was generally more intensive than its response to an equivalent stimulus exerted during the daytime (Fig. 3 a).

\section{Effect of two successive acute stress signals on the HPA system dynamics}

Dynamical response of the HPA axis model to two successive cortisol perturbations is presented in Fig. 4. States $1 \mathrm{a}, 1 \mathrm{~b}$, and 1c (Fig. 4a-c, respectively) were elicited by applying primary perturbations of different intensities at the same ultradian cortisol oscillation (the peak commencing around 10:00 h). The response of the HPA model to the secondary perturbation depended strongly on the state achieved after the primary perturbation (Fig. 4d, e). 
These results demonstrate that the sensitivity of the HPA system to perturbations depends on the history of the system, i.e. on the state dictated by the first perturbation. Furthermore, these results indicate that the amplitude of the perturbed ultradian oscillations is an important determinant of the HPA axis response to stress (Fig. 4e).

\section{Chronic stress}

Chronic stress can be regarded as a physiological and behavioral state of an organism that has emerged as a response to recurrent homeostasis disturbing challenges that persist for weeks, months or longer [42]. An organism may be exposed to chronic stress continuously, or through cascades of inappropriate requirements whose cumulative effect is achieved by the onset of a new stimulus before the negative effect of the previous one has died out. In our previous study, chronic stress was modeled by applying a sequence of $\mathrm{CRH}$ pulses, whose amplitude and time of onset were randomly varied [19]. Intermittent perturbation of the HPA axis dynamics yielded a complex cortisol oscillation pattern, with ultradian oscillations of randomly varying amplitudes and frequencies (Fig. 6 in reference [19]). Different perturbation patterns generated different response patterns, but even though the details were different, one thing was common - chronic stress altered the mean CRH concentration. This is in line with experimental findings showing that chronic stress changes the mean CRH levels [8], triggering further modifications of the HPA axis dynamics, which altogether are often associated with metabolic and psychological impairments $[7,8,43]$. Therefore, we emulate here chronic stress by varying the parameters $\mathrm{k}_{0}, \mathrm{~d}_{1}$ and $\mathrm{d}_{2}$ that define the CRH dynamics in our model. On one hand, an unambiguous relation exists between the parameter $\mathrm{k}_{0}$ and the stable stationary state $\mathrm{CRH}$ concentration $[\mathrm{CRH}]_{\mathrm{SS}}$ (Eq. (6)) and the parameter $d_{1}$ and the mean daily CRH concentration in the hypothalamic-pituitary portal vessels $<$ [CRH $]>$ (Eq. (7)), on the other. Therefore, all results related to chronic stress are presented as a function of the biologically relevant parameters $[\mathrm{CRH}]_{\mathrm{SS}}$ and $<[\mathrm{CRH}]>$. The parameter $\mathrm{d}_{2}$ defines the amplitude of the circadian CRH oscillation (Eq. (5)).

\section{Effect of chronic stress on ultradian oscillations}

The stationary state value of CRH concentration $\left([\mathrm{CRH}]_{\mathrm{SS}}\right)$ has a decisive effect on the ultradian dynamics of the HPA system (Figs. 1a and b). The corresponding bifurcation diagram, showing how the dynamic state of the HPA system changes depending on the control parameter $[\mathrm{CRH}]_{\mathrm{SS}}$ is given in Fig. 5a. In Fig. $5 \mathrm{a}$, the oscillatory states are presented by pairs (minima and maxima) of cortisol concentrations in the ultradian oscillations, whereas single points denote stable stationary states. Obviously, the ultradian cortisol oscillations exist within a defined range of $[\mathrm{CRH}]_{\mathrm{SS}}$ values, whereas stable steady states were observed outside this interval.

\section{Effect of chronic stress on ultradian and circadian dynamics}

In the model with coupled ultradian and circadian rhythms, the mean daily concentration of CRH in the hypothalamic-pituitary portal vessels $(<[\mathrm{CRH}]>)$ represents the chronic stress parameter, since the hormone concentration levels can be changed by chronic stress for longer periods of time or permanently $[4,8]$. Alteration of $<[\mathrm{CRH}]>$ evoked qualitative alterations in the HPA dynamics (Fig. 5b) that were analogous to the bifurcation pattern observed for ultradian oscillations alone (Fig. 5a) - the oscillations existed only within a certain range of $<[\mathrm{CRH}]>$ values, whereas stable steady states were observed outside this interval. However, $<[\mathrm{CRH}]>$ affected the dynamics of cortisol release during the daytime (Fig. 5b, squares) differently than during the night (Fig. 5b, circles).

In addition, bifurcation diagrams shown in Fig. $5 b$ illustrate that the amplitude of ultradian cortisol oscillations initially increases and thereafter decreases as a function of $<[\mathrm{CRH}]>$ as the control parameter. Hence, continuous elevation of $<[\mathrm{CRH}]>$ levels can produce both an increase and a decrease of ultradian cortisol amplitudes and, consequently, opposite responses of the HPA system to stress. This observation has very important implications - as the amplitude of ultradian cortisol oscillations is the measure of the HPA system's capability to protect itself against acute stress, and the $<[\mathrm{CRH}]>$ is the measure of the chronic stress intensity, we can conclude that mild elevation of cortisol levels due to chronic stress may initially exert beneficial effects on the HPA system capacity to cope with acute external perturbations. Still higher stress will reduce the amplitude of the ultradian HPA oscillations, thus reducing the HPA system's capacity to respond to stress. Eventually, the dynamical regulation capacity of the HPA axis may be lost as the system undergoes a transition to a stable steady state. 
a

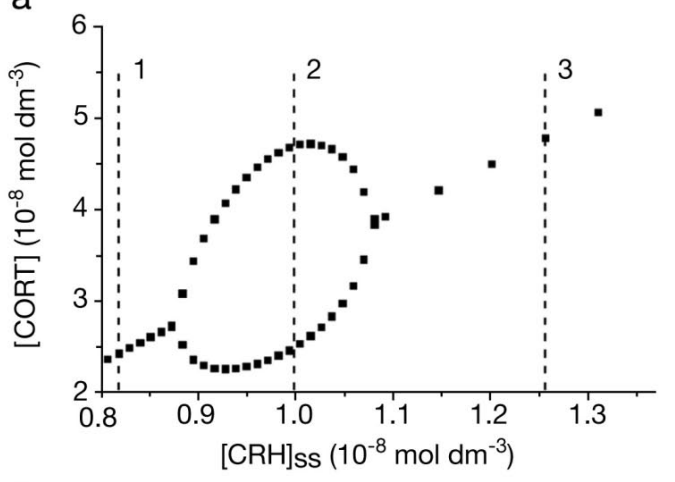

c
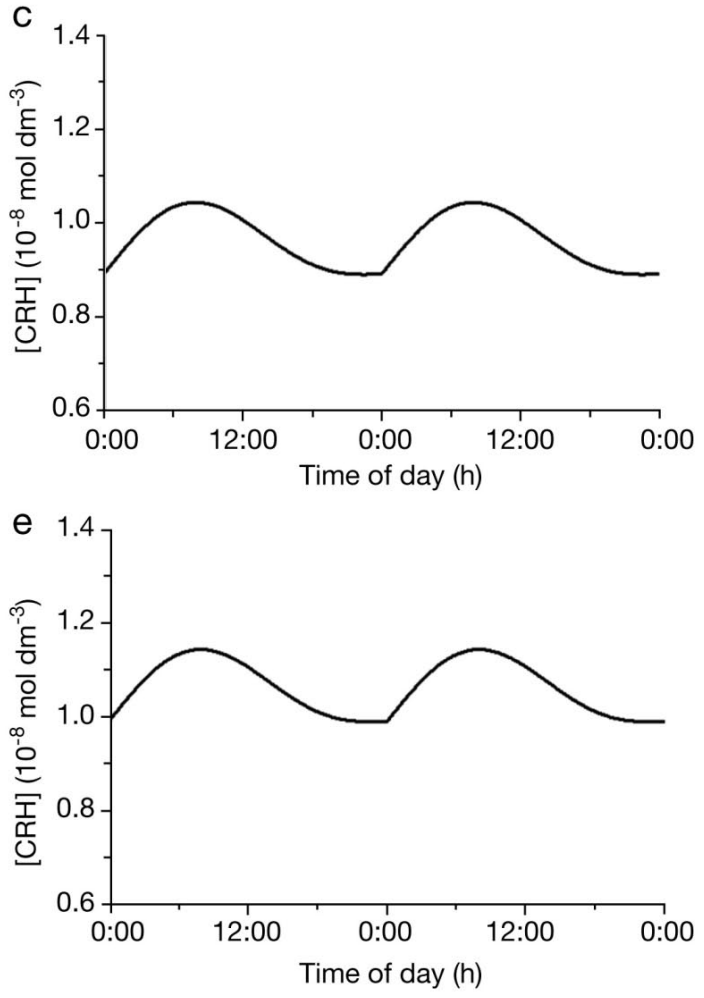

b
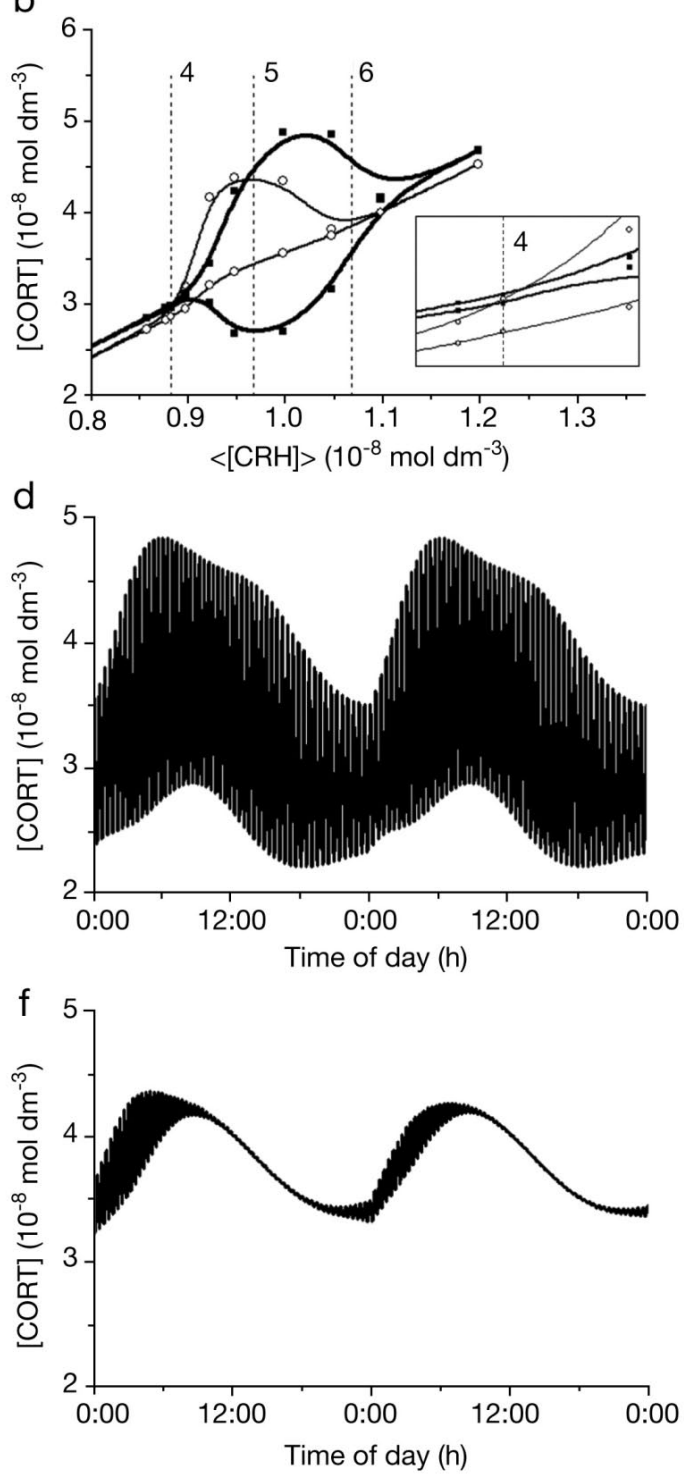

Fig. 5 (a) Bifurcation diagram showing the ultradian cortisol dynamics as a function of $[\mathrm{CRH}]_{\mathrm{SS}}$. Single points denote stable stationary states, whereas the oscillatory states are presented by pairs (minima and maxima) of cortisol concentrations in the ultradian oscillations. Vertical lines indicate three different dynamic states: (1) at low CRH levels, $[\mathrm{CRH}]_{\mathrm{SS}}=0.82 \cdot 10^{-8} \mathrm{~mol} \mathrm{dm}^{-3}$, a low cortisol concentration steady state, denoted as 1 in Fig. $1 \mathrm{~b}$, is established. (2) At $[\mathrm{CRH}]_{\mathrm{SS}}=1.00 \cdot 10^{-8} \mathrm{~mol} \mathrm{dm}^{-3}$, ultradian cortisol oscillations, denoted as 2 in Fig. 1b, are established. (3) At high CRH levels, $[\mathrm{CRH}]_{\mathrm{SS}}=1.26 \cdot 10^{-8} \mathrm{~mol} \mathrm{dm}^{-3}$, a high cortisol concentration steady state, denoted as 3 in Fig. 1b, is established. (b) Bifurcation diagrams showing the ultradian cortisol dynamics as a function of $<[\mathrm{CRH}]>$. In a model with circadian regulation, nocturnal $(\boldsymbol{\square})$ and daytime $(\circ)$ ultradian cortisol oscillations are different. Vertical lines indicate three different dynamic states: (4) at low $<[\mathrm{CRH}]>=0.88524 \cdot 10^{-8} \mathrm{~mol} \mathrm{dm}^{-3}$ (basic physiological conditions), ultradian oscillations are observed during the daytime whereas the amplitude of nocturnal ultradian oscillations is very small (this region is magnified in the insert; corresponding CORT time series are shown in Fig. 1d). (5) At $<[\mathrm{CRH}]>=0.97 \cdot 10^{-8} \mathrm{~mol} \mathrm{dm}^{-3}$, ultradian oscillations with relatively large amplitudes are observed during daytime and at night (corresponding CRH and CORT time series are shown in Fig. $5 \mathrm{c}$ and 5 d, respectively). (6) At $<[\mathrm{CRH}]>=1.07 \cdot 10^{-8}$ mol dm${ }^{-3}$, the ultradian oscillatory dynamics is reversed, showing large-amplitude cortisol oscillations at night, while smallamplitude cortisol oscillations during daytime (corresponding CRH and CORT time series are shown in Fig. 5e and 5f). In all cases, $\mathrm{d}_{2}=0.957$. 

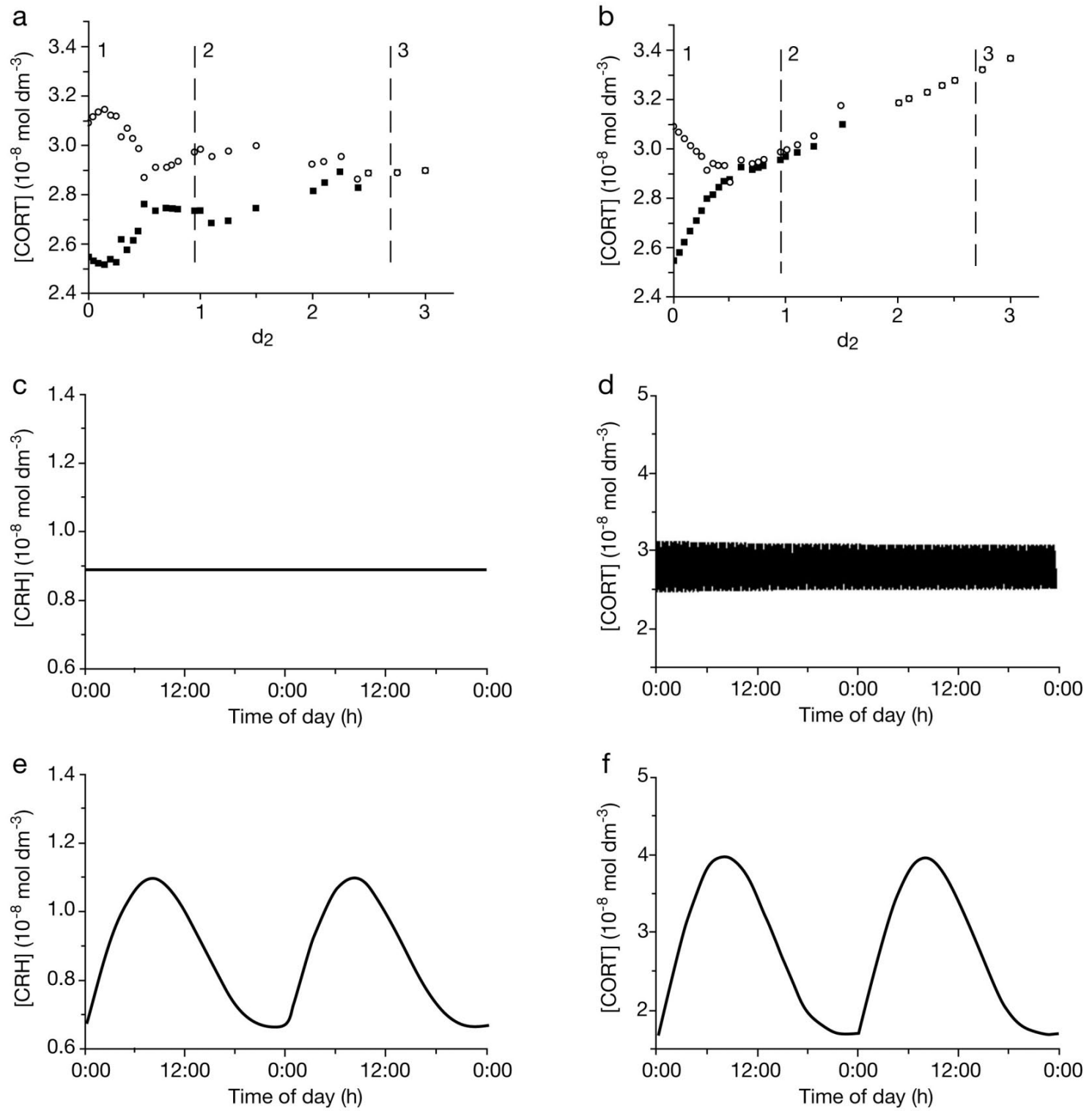

Fig. 6 Cortisol dynamics as a function of the CRH circadian amplitude, modeled via the parameter $\mathrm{d}_{2}$ as the chronic stress determinant.

The bifurcation diagram shows cortisol concentration for a reference ultradian cortisol oscillation during daytime (a) and at night (b). The maximum (open circle) and minimum (filled square) of the ultradian oscillation are indicated. A stationary state is represented by a single symbol (open circle). For $d_{2}=0$, amplitudes of the daytime $\left(A_{\text {day }}(C O R T)\right)$ and nocturnal $\left(A_{\text {night }}(C O R T)\right.$ ) cortisol oscillations have equal values: $A_{\text {day }}(C O R T)=A_{\text {night }}(C O R T)=0.5 \cdot 10^{-8} \mathrm{~mol} \mathrm{dm}^{-3}$. Vertical lines $(1,2$ and 3$)$ depict different states: (1) under ultradian regulation, $\mathrm{d}_{2}=0$, CRH concentration is constant (c) and ultradian cortisol oscillations (d) can be observed. Circadian oscillations under stress exist for high values of $d_{2}\left(d_{2}=2.7\right.$, state 3$)$ for both CRH (e) and cortisol (f), while ultradian cortisol pulses are lost (f). In all cases, $<[\mathrm{CRH}]>=0.88524 \mathrm{~mol} \mathrm{dm}^{-3}$.

Effect of CRH circadian amplitude on the HPA system dynamics

Variation of the circadian amplitude of CRH via the control parameter $\mathrm{d}_{2}$ also affects the HPA system's dynamics (Fig. 6). Parameter $d_{2}$ multiplies time dependent, periodic part of the function $D$ (Eq. 5), therefore governing the amplitude of the extrinsic circadian rhythm. As before (Fig. 5b), the daytime cortisol discharge dynamics (Fig. 6a) was differently affected than its discharge dynamics during the night (Fig. 6b). In the absence of the CRH circadian dive $\left(\mathrm{d}_{2}=0\right)$, $\mathrm{CRH}$ was continuously secreted (Fig. 6c) and the cortisol discharge dynamics showed properties of ultradian secretion (Fig. 6d). By increasing the circadian CRH ampli- 


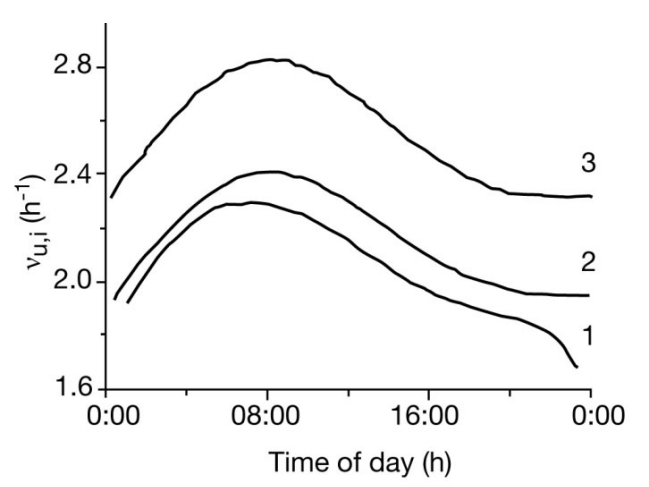

Fig. 7 Effect of the circadian rhythm on the frequency of ultradian cortisol oscillations, $v_{\mathrm{u}, \mathrm{i}}=1 / \mathrm{T}_{\mathrm{u}, \mathrm{i}}$, under basal conditions (1) and chronic stress of different intensity (2 and 3). Depending on the $<[\mathrm{CRH}]>$ value, the ultradian cortisol oscillations frequency $\left(v_{\mathrm{u}, \mathrm{i}}\right)$ and their number over $24 \mathrm{~h}$ $\left(N_{\mathrm{u}}\right)$ are modified: $(1)<[\mathrm{CRH}]>=0.88524 \cdot 10^{-8} \mathrm{~mol} \mathrm{dm}^{-3}$ (basal physiology), $N_{\mathrm{u}}=49$; (2) $<[\mathrm{CRH}]>=0.925 \cdot 10^{-8} \mathrm{~mol}$ $\mathrm{dm}^{-3}$ (chronic stress), $N_{\mathrm{u}}=52$; (3) $<[\mathrm{CRH}]>=1.05 \cdot 10^{-8}$ mol dm ${ }^{-3}$ (chronic stress), $N_{\mathrm{u}}=64$.

tude (that is equivalent to increasing $\mathrm{d}_{2}$ ), the amplitude of the ultradian cortisol oscillation changed (doublet points in Fig. $6 \mathrm{a}$ and $6 \mathrm{~b}$ ), until a point was reached after which the cortisol ultradian oscillatory evolution died out (single points in Fig. 6a and 6b), whereas circadian rhythmicity of both CRH and cortisol was preserved (Fig. 6e and 6f, respectively).

One should notice that Fig. 5a, 5b, 6a, and $6 \mathrm{~b}$ are bifurcation diagrams, showing qualitative changes in the HPA axis dynamics when the control parameter is varied. Together, these bifurcation diagrams show that cortisol discharge dynamics is pulsatile only for certain parameter values $\left(<[\mathrm{CRH}]>, \mathrm{d}_{2}\right)$.

Effect of the chronic stress on the frequency of ultradian oscillations

When uncoupled from the circadian rhythm, the frequency of ultradian cortisol oscillations did not change over time (Fig. 1b). Conversely, in the case of coupling between circadian and ultradian oscillations, ultradian oscillation frequency became time-dependent, showing the highest frequency during the circadian peak (Fig. 7, curve 1).

Chronic stress, modeled as before by varying the mean daily concentration of CRH concentration in the hypothalamic-pituitary portal vessels $(<[\mathrm{CRH}]>)$, increased the frequency of the ultradian oscillations over the 24 h course (Fig. 7, curves 2 and 3).

\section{Discussion}

As expected, cortisol and CRH perturbations inevitably change the dynamics of the examined HPA axis hormones (Figs. 2-7). At present, our model predictions are in quantitative agreement with experimentally measured aldosterone (data not shown) and cortisol levels (Figs. 1-7), whereas for ACTH and CRH, only qualitative agreement is achieved (The concentrations of ACTH and CRH are not yet optimized and deviate from experimentally measured values. This is a consequence of the low-dimensionality of our model - in order to maintain a small number of variables, precursor species are excluded from the present model. Even so, the generality of conclusions reported in this study is not compromised by this discrepancy). Acute stress exerts transient effects on the HPA system dynamics (Figs. 2-4), whereas the effect of chronic stress is longlasting, shifting the whole HPA system to new dynamic states (Figs. 5-7). These newly achieved dynamic states are characterized by different amplitudes, frequencies and the general appearance of the ultradian oscillations of all considered HPA system hormones.

In line with model predictions, according to which the HPA axis response to stress depends on the ultradian phase (Fig. 2), there are several experimental observations showing that acute stress induces different effects depending on the phase of the ultradian secretion pulse at which the stress has been induced [11, 13-15]. For example, it has been found that the degree of prednisolone-induced rapid inhibition of cortisol was greater when the time after prednisolone injection to pulse onset was longer [11]. This indicates that HPA axis responsiveness to perturbations with prednisolone depends on the ultradian phase of the endogenous cortisol oscillations.

Dependence of corticosterone concentration response to acute stress on the phase (descending/ascending) of the ultradian rhythm at the moment the stress commences has also been observed in in vivo experiments with rats [13-15]. It has been shown in these studies that the same acute stress ( 5 min white noise stress of $114 \mathrm{~dB}$ ) applied either in the ascending (secreting) and interpulse phase or during the descending (non-secreting) phase of corticosterone ultradian rhythm evoked significant increase of corticosterone level only when it had been applied during the ascending [13-15] or interpulse [15] phase of ultradian basal corticosterone pulse. No significant response was detected when the 
stressful stimulus was applied during the descending phase [13-15].

There are also converse reports, showing that intravenous injection of specific dosage of CRH $(2 \mu \mathrm{g})$ administered to rats during the rising or falling phase of the corticosterone ultradian oscillations failed to induce significant difference between the two rat groups [14]. A possible explanation may be that the dosage tested is not adequate. HPA response to stress depends not only upon timing, i.e. the phase at which the treatment was applied, but also upon the intensity of the perturbation (Fig. 3). Thus, another CRH dosage could have been more appropriate.

Similarly, it has been demonstrated that cortisol (and ACTH) response to intravenously administered prednisolone did not significantly depend on time of day the administration occurred [11]. However, these experiments were performed in the morning and afternoon, but not during the night. In addition, only one prednisolone dose was tested. Therefore, before ruling out the possibility that diurnal dependence of the HPA axis response to prednisolone (or other externally induced glucocorticoids) exists in humans, additional experiments might be useful.

Due to its long duration and/or frequent incidence, chronic stress may alter receptor localization, rates of chemical reactions and transporting processes in different brain regions, such as the hippocampus, amygdala and prefrontal cortex [44-47], causing altered patterns of activity observable by PET (positron emission tomography), fMRI (functional magnetic resonance imaging), MEG (magnetoencephalography) or EEG (electroencephalography). Under certain conditions the allostasis, i.e. dynamic regulatory mechanisms may no longer be efficient, causing allostatic overload [28, 47]. Such changes may be reflected by the bifurcation diagrams shown in Figs. 5 and 6. Allostatic overload of the HPA system, i.e. the incapability of the HPA axis to cope with the "external pressure" may occur in the form of bifurcation points (Figs. 5 and 6). Transition through a bifurcation point may indicate that the dynamic regulatory mechanism has collapsed, which may be possibly interpreted as a condition that leads to disease onset. Such dynamic modifications may be reversible, but may also be irreversible, showing hysteresis. Thus, even though the stress has ceased, the HPA system does not return to its original physiological state but may end up in a new nonequilibrium stationary state.

The HPA system dynamics is tightly related to sleep- awake behavior. All HPA hormones participate in sleep regulation. Furthermore, manipulation of the sleepwake pattern induces subtle changes in the HPA system dynamics. For example, CRH and ACTH administration have been found to impair sleep [48], whereas chronic insomnia is associated with increased secretion and number of ultradian pulses of cortisol and ACTH during the $24 \mathrm{~h}$ period [49]. Our model correctly predicts that increase in the mean daily concentration of $\mathrm{CRH}$ in the hypothalamic-pituitary portal vessels $(<[\mathrm{CRH}]>)$ alters the HPA system dynamics (Fig. 5), eventually leading to a radical disruption of the ultradian secretion dynamics. Under such extreme conditions, the ultradian secretion becomes more active during night (Fig. 5f) rather than during daytime. This inverse secretory activity, with more pronounced nocturnal cortisol and ACTH pulses, was also observed in patients suffering from chronic insomnia [49].

Bifurcation diagrams shown in Fig. 5 may be regarded as dynamical explanation for the adverse response of the HPA axis to chronic stress, offering a theoretical account for the apparently contradictory observation reported in the literature that chronic stress can yield both hypo- and hypercortisolism [43].

It is also well known that stress induces changes in the immune system function. This modulation is achieved via the effect of glucocorticoid hormones and other components of the HPA axis [46-48, 50]. Bifurcation analysis may be helpful for understanding why chronic stress alters the immune system function distinctly and in a non-linear fashion [41, 51].

\section{Conclusion}

Adaptive transformations in HPA axis dynamics following acute and chronic perturbations were studied using a stoichiometric model of the HPA axis. Model predictions were related with experimental and clinical observations reported in the literature. We outline briefly here the most relevant positive correlations between model predictions and real observations.

As expected, acute stress transiently perturbs the HPA axis dynamics. The response of the HPA axis is complex, depending on the intensity and the time of stress onset (Figs. 2-4). Numerical simulations (Figs. 2,3 ) reproduced the empirically well established notion that the same stimulus applied at slightly different time points, may induce different, even opposite changes in cortisol levels in the same individual [13-15]. 
Modeling elucidates why biphasic dynamic regulation of the HPA axis activity, achieved through the coupling of ultradian and circadian regulation of HPA hormone release, is an efficient strategy for stress modulation, providing at the same time robustness and plasticity. Modeling also reveals the boundaries under which this regulatory mechanism is operational (Figs. 5 and 6). Numerical simulations clarify why stressinduced increase in mean daily CRH levels in the hypothalamic-pituitary portal vessels (control parameter $<$ [CRH] $>$ in the model) may increase or decrease mean cortisol levels, showing that there is no contradiction in such observations. In this respect, modeling could serve as theoretical background to answer how and under which circumstances chronic stress leads to hyper- or hypocortisolism, opposite states observed in stress-related illnesses such as major depression or posttraumatic stress disorder [43].

Numerical simulations also reveal that ultradian cortisol dynamics may be reversed under chronic stress, causing more active hormone release during the night instead of daytime (Fig. 5 f). Such changes in the HPA axis secretory activity may be the primary cause of an array of changes potentially leading to insomnia [49].

Modeling predicts that chronic stress initially increases the amplitude of ultradian cortisol oscillations (Fig. 5a, b). This effect may be beneficial at first - as the amplitude is increased, the sensitivity of the HPA axis to acute stress is reduced. Hence, the increasing amplitude of the ultradian cortisol pulsation may act as a protective, tolerance mechanism. Such regulatory mechanism may also explain why we are best fit for interactions/activity during a certain part of the day.

Modeling also predicts that qualitative changes in the HPA axis dynamics may occur under chronic stress (Fig. 5d, f). Such global changes in the HPA axis dynamics may explain how allostatic overload occurs and why under certain conditions, the dynamic regulatory mechanism is not turned on, or why a certain response is not turned off when it is no longer needed.

\section{Appendix}

\section{Using modeling as a tool for designing glucocorticoid pharmacotherapy distribution strategies}

Our results imply that the effect of pharmacotherapy may depend on therapy distribution strategies in a complex fashion. However, our results also suggest that even very intricate behavior of the HPA axis is deterministic in nature, stemming from the intrinsic rhythmicity of the HPA axis [10] and can therefore be modeled and predicted. This means that insights on HPA axis dynamics that are revealed by modeling may be used as cues for designing treatment strategies. In that regard, we wish to address the significance of timing in glucocorticoid therapy.

Significance of circadian timing for administration of glucocorticoid therapy has been acknowledged in patients suffering from asthma [52] or Addison's disease [53]. Novel strategies of delayed and sustained cortisol release developed for the treatment of these conditions are expected to mimic the circadian cortisol rhythm more accurately, thus reducing the side-effects of the therapy $[54,55]$. In rodents, an infusion protocol capable of mimicking corticosterone ultradian rhythm has been developed, enabling ultradian control of corticosterone amplitude and frequency in adrenalectomized animals [10]. Some of these aspects have been also recognized in the alternate day therapy with glucocorticoids, where empirical data show that the application of the same dose of glucocorticoids every second morning is more beneficial to patients than if given every day [32, 56, 57].

\section{The concept of dynamics management}

The complexity of the HPA axis dynamics requires a comprehensive approach when treating its malfunctions. Alongside with gene manipulation or development of novel, specific drugs, additional avenue emerges, in which the dynamics of the HPA axis is supposed to be manipulated in a relatively simple manner, by stressing the system at the right time (phase angles of oscillations) with the right concentration(s) of perturbing internal species (glucocorticoids, CRH, ACTH, aldosterone etc.). We refer to this approach of controlled change of dynamics towards desired direction by utilizing appropriate perturbation as dynamics management. This approach could help redesigning the existing therapeutic procedures (e.g. glucocorticoid administrations), making them more efficient and with less side-effects. If a patient's condition allows for, before starting any therapy, a screening of the patient's HPA axis dynamics should be made with high-enough resolution in order for the ultradian oscillations to be sufficiently characterized. This could be particularly important bearing in mind the interpersonal and intrapersonal differences in the HPA axis dynamics [29, 30], i.e. the fact that the initial state of the axis at the time 
of the onset of the therapy is very dissimilar, not only among different individuals, but as well within the same individual over the course of time.

\section{Significance of the initial state of the HPA axis}

The initial dynamical state of the HPA axis during the onset of treatment is a major determinant of the HPA systems response to the applied treatment. In medical treatments with glucocorticoids, these drugs do not necessarily have the same effects when applied to patients in different physiological states, such as milder form of infection, or the state of acute shock. In the first case, well-established doses of glucocorticoids exert their inhibitory effect on cortisol levels, but in the latter, they could fail in doing so. In a case-study on patients with hypercortisolism evoked by the state of circulatory (septic or non-septic) shock, intravenous infusion of dexamethasone failed to suppress cortisol concentration elevated due to circulatory shock, in contrast with the control group of healthy subjects, where dexamethasone infusion exerted its common behavior - the complete suppression of cortisol [58]. Presumably, the circulatory shock induced alteration of the HPA axis dynamics, shifting the axis to a novel non-basal dynamical state, so when the therapy with dexamethasone had been administered, the axis was unable to exert the same effect (suppression) as it does in basal conditions. Given the results presented in this paper, there probably exists a specific dosage of dexamethasone, different than that for basal dynamical state, at which the cortisol suppression within the HPA axis of non-basal dynamics can occur.

The unique interrelation between the initial state and subsequent dynamical HPA response to perturbations can also be employed for making differential diagnoses. A good example is the low-dose dexamethasone-
CRH stimulation test, a diagnostic test clinically used to distinguish patients with Cushing's syndrome from those of pseudo-Cushing's state [59]. In this study, two groups of patients responded differentially to the test: low-dose dexamethasone administration (low-dose dexamethasone suppression test) suppressed cortisol and ACTH levels in patients with pseudo-Cushing's states to a larger extent than in those with Cushing's syndrome, while the Cushing's syndrome patients showed greater response (cortisol and ACTH elevation) than the ones with pseudo-Cushing's states to the subsequent $\mathrm{CRH}$ administration (CRH stimulation test). Combined, the two tests (two perturbations) evoked a unique response within the two patient groups, distinctive enough to set the criterion for discrimination of the two dysfunctions. Thus, although both conditions are associated with hypercortisolism, their original pathophysiology [60] i.e. initial states are dissimilar, and this difference was reflected in the low-dose dexamethasone-CRH stimulation test with very high specificity, sensitivity and diagnostic accuracy.

As the understanding of the complexity of the HPA axis increases and experimental procedures for tracking and modifying its activity in time advance, it might even be possible to make personal "phase diagrams" of HPA axis response to stress. These diagrams could be used as indicators for designing the most appropriate therapy for each individual patient.

\section{Acknowledgements}

Financial support from the Ministry of Science and Technological Development of Serbia, grants no. 172015 and 45001, the Swedish Research Council, The Swedish Brain Foundation and the Knut and Alice Wallenberg Foundation is gratefully acknowledged.

\section{References}

1. Goldbetter A (1996), Biochemical Oscillations and Cellular Rhythms. Cambridge University Press, Cambridge.

2. Koukkari WL, Sothern RB, Introducing biological rhythms. Springer Science Business Media Inc., New York.

3. Lightman SL, Windle RJ, Ma XM, Harbuz MS, Shanks NM, Julian MD, Wood SA, Kershaw YM, Ingram CD (2002) Hypothalamic-pituitary-adrenal function. Arch Physiol Biochem 110: 90-93.
4. Miller WL, Chrousos GP (2001) The Adrenal Cortex. In Felig P, Frohman L (eds) Endocrinology \& Metabolism. McGraw-Hill, New York: 387-524.

5. Tsigos C, Chrousos GP (2002) Hypothalamic-pituitaryadrenal axis, neuroendocrine factors and stress. $J$ Psychosom Res 53: 865-871.

6. Hartmann A, Veldhuis JD, Deuschle M, Standhardt H, Heuser I (1997) Twenty-four hour cortisol release profiles in patients with Alzheimer's and Parkinson's disease compared to normal controls: ultradian secretory 
pulsatility and diurnal variation. Neurobiol Aging 18: 285-289.

7. Nader N, Chrousos GP, Kino T (2010) Interactions of the circadian CLOCK system and the HPA axis. Trends Endocrinol Metab 21: 277-286.

8. Swaab DF, Bao AM, Lucassen PJ (2005) The stress system in the human brain in depression and neurodegeneration. Ageing Res Rev 4: 141-194.

9. Conway-Campbell BL, McKenna MA, Wiles CC, Atkinson HC, De Kloet ER, Lightman SL (2007) Proteasome-dependent down-regulation of activated nuclear hippocampal glucocorticoid receptors determines dynamic responses to corticosterone. Endocrinology 148: 5470-5477.

10. Lightman SL, Wiles CC, Atkinson HC, Henley DE, Russell GM, Leendertz JA, McKenna MA, Spiga F, Wood SA, Conway-Campbell BL (2008) The significance of glucocorticoid pulsatility. Eur J Pharmacol 583: 255-262.

11. Russell GM, Henley DE, Leendertz J, Douthwaite JA, Wood SA, Stevens A, Woltersdorf WW, Peeters BW, Ruigt GS, White A, Veldhuis JD, Lightman SL (2010) Rapid glucocorticoid receptor-mediated inhibition of hypothalamic-pituitary-adrenal ultradian activity in healthy males. J Neurosci 30: 6106-6115.

12. Stavreva DA, Wiench M, John S, Conway-Campbell BL, McKenna MA, Pooley JR, Johnson TA, Voss TC, Lightman SL, Hager GL. (2009). Ultradian hormone stimulation induces glucocorticoid receptor-mediated pulses of gene transcription. Nat Cell Biol 11: 10931102.

13. Windle RJ, Wood SA, Shanks N, Lightman SL, Ingram CD (1998) Ultradian rhythm of basal corticosterone release in the female rat: dynamic interaction with the response to acute stress. Endocrinology 139: 443-450.

14. Windle RJ, Wood SA, Lightman SL, Ingram CD (1998) The pulsatile characteristics of hypothalamo-pituitaryadrenal activity in female Lewis and Fischer 344 rats and its relationship to differential stress responses. Endocrinology 139: 4044-4052.

15. Windle RJ, Wood SA, Kershaw YM, Lightman SL, Ingram CD, Harbuz MS (2001) Increased corticosterone pulse frequency during adjuvant-induced arthritis and its relationship to alterations in stress responsiveness. J Neuroendocrinol 13: 905-911.

16. Bairagi N, Chatterjee S, Chattopadhyay J (2008) Variability in the secretion of corticotropin-releasing hormone, adrenocorticotropic hormone and cortisol and understandability of the hypothalamic-pituitary-adrenal axis dynamics - a mathematical study based on clinical evidence. Math Med Biol 25: 37-63.

17. Jelić S, Čupić Ž, Kolar-Anić Lj (2005) Mathematical modelling of the hypothalamic-pituitary-adrenal system activity. Math Biosci 197: 173-187.

18. Jelić S, Čupić Ž, Kolar-Anić Lj (2008) Modelling of the hypothalamic-pituitary-adrenal system activity based on the stoichiometric analysis. In Romano E, De Luca S (eds) New research on neurosecretory systems. Nova Science Publishers Inc., New York: 225-245.

19. Jelić S, Čupić Ž, Kolar-Anić Lj, Vukojević V (2009) Predictive modeling of the hypothalamic-pituitary-adrenal (HPA) function. Dynamic system theory approach by stochiometric network analysis and quenching of small amplitude oscillations. Int J Nonolin Sci Num 10: 1451-1472.

20. Keenan DM, Licinio J, Veldhuis JD (2001) A feedbackcontrolled ensemble model of the stress-responsive hypothalamo-pituitary-adrenal axis. Proc Natl Acad Sci USA 98: 4028-4033.

21. Keenan DM, Veldhuis JD (2003) Cortisol feedback state governs adrenocorticotropin secretory-burst shape, frequency, and mass in a dual-waveform construct: time of day-dependent regulation. Am J Physiol Regul Integr Comp Physiol 285: R950-R961.

22. Keenan DM, Roelfsema F, Veldhuis, JD (2004) Endogenous ACTH concentration-dependent drive of pulsatile cortisol secretion in the human. Am J Physiol Endocrinol Metab 287: E652-E661.

23. Keenan DM, Chattopadhyay S, Veldhuis JD (2005) Composite model of time-varying appearance and disappearance of neurohormone pulse signals in blood. $J$ Theor Biol 236: 242-255.

24. Lenbury Y, Pornsawad P (2005) A delay-differential equation model of the feedback-controlled hypothalamus-pituitary-adrenal axis in humans, Math Med Biol 22: $15-33$.

25. Marković V, Jelić S, Vukojević V, Kolar-Anić Lj (2010) Modelling the hypothalamic-pituitary-adrenal (HPA) axis response to external perturbations with cortisol. Physical Chemistry 2010. Society of Physical Chemists of Serbia, Belgrade: 212-214.

26. Veldhuis JD, Keenan DM, Pincus SM (2008) Motivations and methods for analyzing pulsatile hormone secretion. Endocr Rev 29: 823-864.

27. Walker JJ, Terry JR, Lightman SL (2010) Origin of ultradian pulsatility in the hypothalamic-pituitary-adrenal axis. Proc $R$ Soc B 277: 1627-1633.

28. McEwen BS (2007) Physiology and neurobiology of stress and adaptation: central role of the brain. Physiol Rev 87: 873-904.

29. Kudielka BM, Hellhammer DH, Wüst S (2009) Why do we respond so differently? Reviewing determinants of human salivary cortisol responses to challenge. Psychoneuroendocrinology 34: 2-18.

30. Kudielka BM, Wüst S (2010) Human models in acute and chronic stress: assessing determinants of individual hypothalamus-pituitary-adrenal axis activity and reactivity, Stress 13: 1-14.

31. VanItallie TB (2002) Stress: a risk factor for serious illness. Metabolism 51: 40-45. 
32. Chrousos GP (2001) Glucocorticoid Therapy. In Felig P, Frohman LA (eds) Endocrinology \& Metabolism. McGraw-Hill, New York: 609-632.

33. Holsboer F (2000) The stress hormone system is back on the map. Curr Psychiatry Rep 2: 454-456.

34. Kellendonk C, Gass P, Kretz O, Schutz G, Tronche F (2002) Corticosteroid receptors in the brain: gene targeting studies. Brain Res Bull 57: 73-83.

35. Makino S, Hashimoto K, Gold PW (2002) Multiple feedback mechanisms activating corticotropinreleasing hormone system in the brain during stress. Pharmacol Biochem Behav 73: 147-158.

36. Schulkin J, Gold PW, McEwen BS (1998) Induction of corticotrophin-releasing hormone gene expression by glucocorticoids: implication for understanding the states of fear and anxiety and allostatic load. Psychoneuroendocrinology 23: 219-243.

37. Benson SW (1960) The Foundations of Chemical Kinetics. McGraw-Hill Book Company, New York: 1960.

38. Yeremin EN (1979) The foundations of Chemical Kinetics. Mir Publishers, Moscow: 1979.

39. Gear CW (1971) Numerical initial value problems in ordinary differential equations. Prentice-Hall Inc., Englewood Cliffs, New Jersey.

40. Engler D, Pham T, Liu JP, Fullerton MJ, Clarke IJ, Funder JW (1990) Studies of the regulation of the hypothalamic-pituitary-adrenal axis in sheep with hypothalamic-pituitary disconnection.II. Evidence for in vivo ultradian hypersecretion of proopiomelanocortin, peptides by the isolated anterior and intermediate pituitary. Endocrinology 127: 1956-1966.

41. Dhabhar FS (2002) Stress-induced augmentation of immune function - the role of stress hormones, leukocyte trafficking, and cytokines. Brain Behav Immun 16: 785-798.

42. Sorrells SF, Caso JR, Munhoz CD, Sapolsky RM (2009) The stressed CNS: when glucocorticoids aggravate inflammation. Neuron 64: 33-39.

43. Miller GE, Chen E, Zhou ES (2007) If it goes up, must it come down? Chronic stress and the hypothalamicpituitary-adrenocortical axis in humans. Psychol Bull 133: 25-45.

44. De Kloet ER, Joëls M, Holsboer F (2005) Stress and the brain: from adaptation to disease. Nat Rev Neurosci 6: 463-475.

45. Holsboer F (2000) The corticosteroid receptor hypothesis of depression. Neuropsychopharmacology 23: 477501.

46. Joëls M., Tallie BZ (2009) The neuro-symphony of stress. Nat Rev Neurosci 10: 459-466.
47. McEwen BS (2008) Central effects of stress hormones in health and disease: understanding the protective and damaging effects of stress and stress mediators. Eur J Pharmacol 583: 174-185.

48. Blaustein JD (2007) Behavioral Neurochemistry and Neuroendocrinology. In Lajtha A (ed) Handbook of Neurochemistry and Molecular Neurobiology. Springer, New York.

49. Vgontzas AN, Bixler EO, Lin HM, Prolo P, Mastorakos G, Vela-Bueno A, Kales A and Chrousos GP (2001) Chronic Insomnia Is Associated with Nyctohemeral Activation of the Hypothalamic-Pituitary-Adrenal Axis: Clinical Implications. J Clin Endocrinol Metab 86: 3787-3794.

50. Sapolsky RM, Romero LM, Munck AU (2000) How do glucocorticoids influence stress responses? Integrating permissive, suppressive, stimulatory, and preparative actions, Endocr Rev 21: 55-89.

51. Martin LB (2009) Stress and immunity in wild vertebrates: timing is everything. Gen Comp Endocrinol 163: 70-76.

52. Reinberg A, Smolensky MH, D’Alonzo GE, McGovern JP (2003) Chronobiology and asthma. III. Timing corticotherapy to biological rhythms to optimize treatment goals. J Asthma 25: 219-248.

53. Chakera AJ, Vaidya B (2010) Addison disease in adults: diagnosis and management. Am. J Med 123: 409-413.

54. Løvås K, Husebye ES (2008) Replacement therapy for Addison's disease: recent developments. Expert Opin Investig Drugs 17: 497-509.

55. Reisch N, Arlt W (2009) Fine tuning for quality of life: 21st century approach to treatment of Addison's disease. Endocrinol Metab Clin North Am 38: 407-418.

56. Fauci AS (1978) Alternate-day corticosteroid therapy. Am J Med, 64: 729-731.

57. Seth A, Aggarwal A (2004) Monitoring adverse reactions to steroid therapy in children. Indian Pediatr 41: 349-357.

58. Perrot D, Bonneton A, Dechaud H, Motin J, Pugeat M (1993) Hypercortisolism in septic shock is not suppressible by dexamethasone infusion. Crit Care Med 21: 396401.

59. Yanovski JA, Cutler GB Jr, Chrousos GP, Nieman LK (1993) Corticotropin-releasing hormone stimulation following low-dose dexamethasone administration: a new test to distinguish Cushing's syndrome from pseudo-Cushing's states. JAMA 269: 2232-2238.

60. Newell-Price J, Trainer P, Besser M, Grossman A (1998) The diagnosis and differential diagnosis of Cushing's syndrome and pseudo-Cushing's states. Endocr Rev 19: 647-672. 